Pacific Journal of Mathematic 


\title{
GENERATING LARGE INDECOMPOSABLE CONTINUA
}

\author{
MiCHEL SMITH
}

\begin{abstract}
It has been shown by D. P. Bellamy that every metric continuum is homeomorphic to a retract of some metric indecomposable continuum. This result was later extended by G. R. Gordh who proved a similar theorem in the non-metric case. In the present paper a different technique is used to generate such continua.
\end{abstract}

It is shown that if $\alpha$ is an infinite cardinal number then there is an indecomposable continuum with $2^{\alpha}$ composants and if $I$ a (non-metric) continuum then $I$ is homeomorphic to a retract of such a continuum. An indecomposable continuum is constructed such that if $C$ is a composant of it and $H$ is an infinite subset of $C$ then $C$ contains a limit point of $H$. Finally a non-metric continuum is found so that each proper subcontinuum of it is metric.

Definitions and notations. A continuum is a compact connected Hausdorff space. Suppose $A$ is a well ordered set, for each $a \in A M_{a}$ is a topological space, and if $a<b$ in $A \theta_{a}^{b}$ is a continuous function from $M_{b}$ onto $M_{a}$ so that if $a<b<c$ in $A$ then $\theta_{a}^{b} \circ \theta_{b}^{c}=\theta_{a}^{c}$. The space $M$ is the inverse limit $M=\lim _{\leftarrow}\left\{M_{a}, \theta\right\}_{a \in A}$ means that $M$ is the topological space to which the point $P$ belongs if and only if $P$ is a function from $A$ into $\bigcup_{a \in A}\left\{M_{a}\right\}$ so that $P_{a} \in M_{a}$ and if $a<b$ in $A$ then $\theta_{a}^{b}\left(P_{b}\right)=P_{a} . \quad R$ is a region in $M$ means that there is an element $a \in A$ and an open set $S \subseteq M_{a}$ so that $R=\left\{P \mid P_{a} \in S\right\}$. $\mathbf{P}_{a}$ denotes the function from $M$ into $M_{a}$ so that $\mathbf{P}_{a}(P)=P_{a}$. If $S=\Pi_{a \in A} S_{a}$ is a product space then $x=\left\{x_{a}\right\}_{a \in A}$ denotes the point of $S$ so that $x_{a} \in S_{a}$, and $\pi_{a}$ denotes the function from $S$ into $S_{a}$ so that $\pi_{a}(x)=x_{a}$. If $\alpha$ is an ordinal number $\Pi_{i<\alpha}[0,1]_{i}$ denotes the cartesian product of $\alpha$ copies of the interval $[0,1]$. If $M=\lim \left\{M_{a}, \theta\right\}_{a \in A}$ and for each $a \in A M_{a}$ is a continuum then $M$ is a continuum. Also if for each $a \in A, M_{a}$ is an indecomposable continuum then so is $M$. For theorems concerning inverse limits the reader should consult [2].

THEOREM 1. Suppose $M$ is a compact continuum, $\alpha$ is a well ordered set with no last element, $M$ is the inverse limit $M=\lim \left\{M_{a}, \theta\right\}_{a \in \alpha}$ of $a$ collection of Hausdorff continua, and for each $a \in \alpha$ there is a subcontinuum $I_{a}$ of $M_{a}$ so that:

(1) $\theta_{a}^{b}\left(I_{b}\right)=M_{a}$ for $a<b$ in $\alpha$, and 

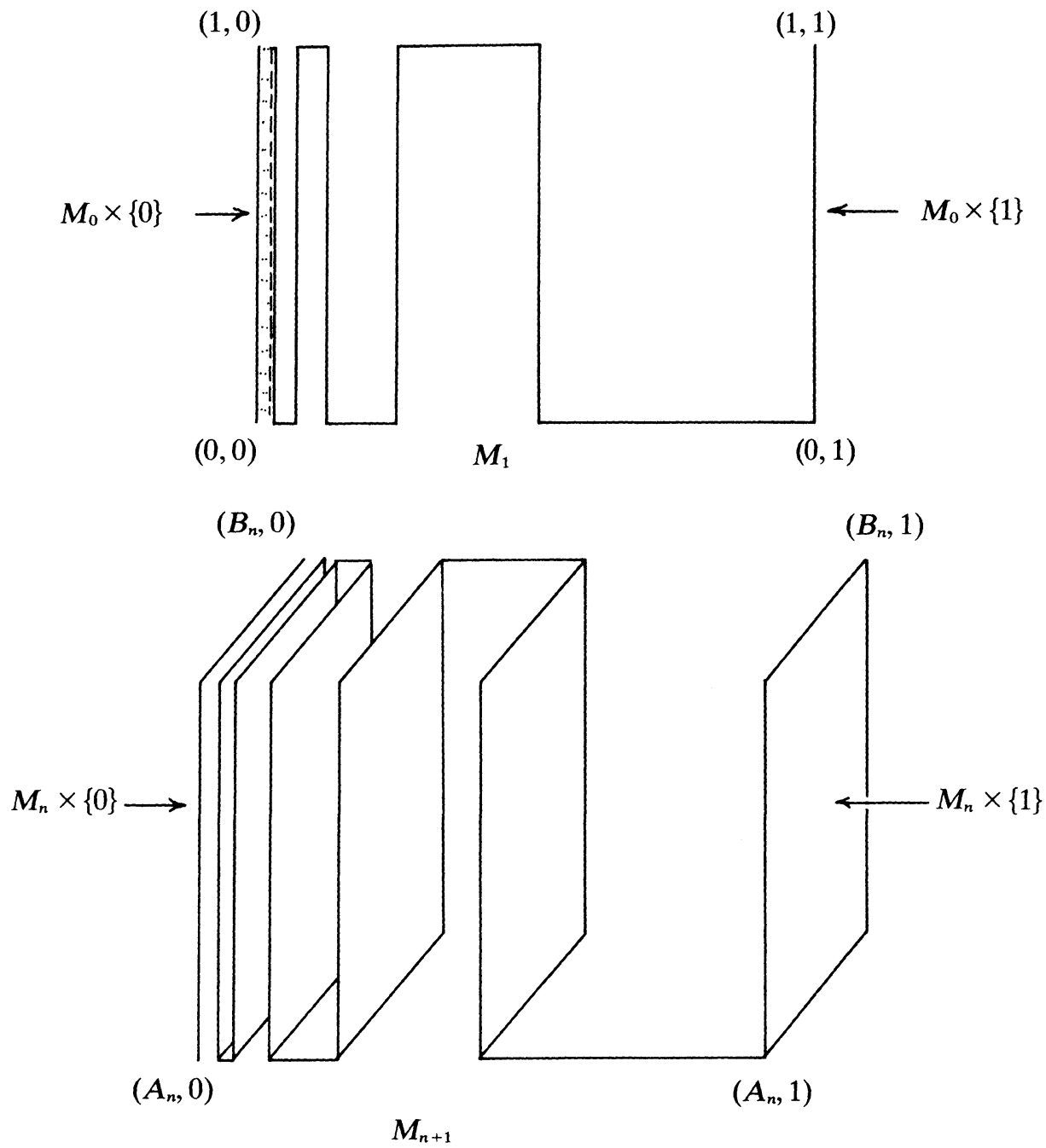

(2) if $I$ is a subcontinuum of $M_{a}$ intersecting $I_{a}$ and $M_{a}-I_{a}$ then $I$ contains $I_{a}$.

Then $M$ is indecomposable.

Proof. Suppose $a \in \alpha$ and $P$ is a point of $M_{a}-I_{a}$. Then there is a subcontinuum $V$ of $M_{a}$ which is irreducible from the point $P$ to $I_{a}$. The set $V-I_{a} \cap V$ is connected and $\overline{V-I_{a} \cap V}=V$. From condition (2) it follows that $I_{a} \subseteq V$, so $I_{a} \subset V=\overline{V-I_{a} \cap V} \subset \overline{M-I_{a}}$.

Now suppose $M$ is the union of two proper subcontinua $H$ and $K$. Let $P$ be a point of $H$ not in $K$ and let $Q$ be a point of $K$ not in $H$. There exists an element $a \in \alpha$ and mutually exclusive regions $R_{a}$ and $S_{a}$ of $M_{a}$ containing $P_{a}$ and $Q_{a}$ respectively so that $R=\left\{x \mid x_{a} \in R_{a}\right\}$ 
does not intersect $K$ and $S=\left\{x \mid x_{a} \in S_{a}\right\}$ does not intersect $H$. Thus $R$ and $S$ are mutually exclusive open sets in $M$ containing $P$ and $Q$ respectively. It follows from the above and condition (1) that $\theta_{a}^{(a+1)^{-1}}\left(R_{a}\right)$ and $\theta_{a}^{(a+1)^{-1}}\left(S_{a}\right)$ are mutually exclusive open sets in $M_{a+1}$ and each intersects both $I_{a+1}$ and $M_{a+1}-I_{a+1}$. So $\mathbf{P}_{a+1}(H)$ and $\mathbf{P}_{a+1}(K)$ both intersect $I_{a+1}$ and $M_{a+1}-I_{a+1}$. So by condition (2) $I_{a+1}$ is a subset of both $\mathbf{P}_{a+1}(H)$ and $\mathbf{P}_{a+1}(K)$. But then $\mathbf{P}_{a}(K)=M_{a}=\mathbf{P}_{a}(H)$, since $\mathbf{P}_{a}=\theta_{a}^{a+1} \circ \mathbf{P}_{a+1}$, which is a contradiction. Thus $M$ is indecomposable.

THEOREM 2. If $q$ is an infinite cardinal number, there is an indecomposable continuum $M$ with $2^{q}$ composants.

Proof. Let $\alpha$ be the first ordinal number so that $|\alpha|=q$. The continuum $M$ will be constructed as an inverse limit of $\alpha$ irreducible continua. Let $M_{0}=[0,1]$. Let $M_{1}$ be the subcontinuum of $[0,1] \times[0,1]$ so that

$$
\begin{aligned}
M_{1}= & \left(M_{0} \times\{0\}\right) \cup\left(\bigcup_{i=0}^{\infty}\left[\left(M_{0} \times\left\{\frac{1}{2 i+1)}\right\}\right) \cup\left(\{0\} \times\left[\frac{1}{2 i+2}, \frac{1}{2 i+1}\right]\right)\right]\right) \\
& \cup\left(\bigcup_{i=1}^{\infty}\left[\left(M_{0} \times\left\{\frac{1}{2 i}\right\}\right) \cup\left(\{1\} \times\left[\frac{1}{2 i+1}, \frac{1}{2 i}\right]\right)\right]\right) .
\end{aligned}
$$

The continuum $M_{1}$ is the union of countably many copies of $M_{0}$ and countably many arcs. If $A_{1}=(0,0)$ and $B_{1}=(1,1)$ then $M_{1}$ is irreducible from $A_{1}$ to $B_{1}$. Let $\theta_{0}^{1}$ be the function from $M_{1}$ onto $M_{0}$ so that $\theta_{0}^{1}\left(P_{1}, P_{2}\right)=P_{1}$.

Suppose that $b<\alpha$ and that $M_{a}$ and $\theta_{c}^{a}$ have been defined for $c<a<b$ so that $M_{a}$ is a subcontinuum of $\Pi_{i \leqq a}[0,1]_{i}$ which is irreducible from the point $A_{a}=\{0\}_{ı}$ to the point $B_{a}=\{1\}_{ı \leqq a}$, and $\theta_{c}^{a}$ is a function from $M_{a}$ onto $M_{c}$ so that $\theta_{c}^{a}\left(\left\{x_{i}\right\}_{i \leqq a}\right)=\left\{x_{i}\right\}_{i \leqq c}$. Suppose that $b$ is not a limit ordinal, $b=a+1$ for some $a<\alpha$. Let $M_{b}$ be the subcontinuum of $\Pi_{i \geqq b}[0,1]$, so that

$$
\text { [*] } \begin{aligned}
M_{b}= & \left(M_{a} \times\{0\}\right) \cup\left(\bigcup _ { i = 0 } ^ { \infty } \left[\left(M_{a} \times\left\{\frac{1}{2 i+1}\right\}\right)\right.\right. \\
& \left.\left.\cup\left(\{A\} \times\left[\frac{1}{2 i+2}, \frac{1}{2 i+1}\right]\right)\right]\right) \\
& \cup\left(\bigcup_{i=1}^{\infty}\left[\left(M_{a} \times\left\{\frac{1}{2 i}\right\}\right) \cup\left(\left\{B_{a}\right\} \times\left[\frac{1}{2 i+1}, \frac{1}{2 i}\right]\right)\right]\right) .
\end{aligned}
$$

The continuum $M_{b}$ is the union of countably many copies of $M_{a}$ and countably many arcs. $\quad M_{b}$ is irreducible from any point of $\left(M_{a} \times\{0\}\right)$ to 
the point $\left(B_{a} \times\{1\}\right)$. Let $A_{b}=A_{a} \times\{0\}$ and $B_{b}=B_{a} \times\{1\}$. Let $\theta_{a}^{b}$ be the function from $M_{b}$ onto $M_{a}$ so that if $\left\{x_{i}\right\}_{i \leqq b} \in M_{b}$ then $\theta_{a}^{b}\left(\left\{x_{i}\right\}_{1 \leqq b}\right)=\left\{x_{i}\right\}_{i \leqq a}$. If $c<a$ define $\theta_{c}^{b}$ to be the function $\theta_{a}^{b} \circ \theta_{c}^{a}$.

Suppose that $b$ is a limit ordinal. Let $M_{b}^{\prime}$ be the continuum $M_{b}^{\prime}=\lim \left\{M_{a}, \theta\right\}_{a<b}$. Let $A_{b}^{\prime}$ denote the point $P$ so that $\mathbf{P}_{a}(P)=A_{a}$ and let $B_{b}^{\prime}$ denote the point $P$ so that $\mathbf{P}_{a}(P)=B_{a}$. Then $M_{b}^{\prime}$ is irreducible from $A_{b}^{\prime}$ to $B_{b}^{\prime}$ since for each $a<b M_{a}$ is irreducible from $\mathbf{P}_{a}\left(A_{b}^{\prime}\right)$ to $\mathbf{P}_{a}\left(B_{b}^{\prime}\right)$. Let $L_{b}$ denote the function from $M_{b}^{\prime}$ into $\Pi_{i<b}[0,1]_{i}$ so that if $P \in M_{b}^{\prime}$ then $L_{b}(P)=\left\{\pi_{l}\left(P_{i}\right)\right\}_{i<b}$ where $P_{i}$ is the $i$ th coordinate of the point $P$, $P_{i}=\mathbf{P}_{t}(P)$. Note that $\mathbf{P}_{i}(P) \in M_{i} \subset \Pi_{k \leqq i}[0,1]_{k} . L_{b}$ is a homeomorphism because if $P$ is a point of $M_{b}^{\prime}$ and $i<j<b$ then $\pi_{a}\left(\mathbf{P}_{t}(P)\right)=\pi_{a}\left(\mathbf{P}_{j}(P)\right)$ for all $a \leqq i$; in other words the ath coordinate in the cartesian product $\Pi_{k \leqq l}[0,1]_{k}$ of $\mathbf{P}_{\iota}(P)$ is the same as the ath coordinate in $\Pi_{k \leqq l}[0,1]_{k}$ of $\mathbf{P}_{i}(P)$. Then $L_{b}\left(M_{b}^{\prime}\right) \subset \Pi_{k<b}[0,1]_{k} . M_{b}$ is defined by replacing $M_{a}$ by $L_{b}\left(M_{b}^{\prime}\right)$ in [*] above and $A_{a}$ by $L_{b}\left(A_{b}^{\prime}\right)$ and $B_{a}$ by $L_{b}\left(B_{b}^{\prime}\right)$. So $M_{b}$ is irreducible from any point of $\left(L_{b}\left(M_{b}^{\prime}\right) \times\{0\}\right)$ to the point $\left(L_{b}\left(B_{b}^{\prime}\right) \times\{1\}\right)$. Let $A_{b}=\left(L_{b}\left(A_{b}^{\prime}\right) \times\{0\}\right)$ and $B_{b}=\left(L_{b}\left(B_{b}^{\prime}\right) \times\{1\}\right)$. If $a<b$ let $\theta_{a}^{b}$ be the function from $M_{b}$ onto $M_{a}$ so that if $\left\{x_{i}\right\}_{i \leqq b} \in M_{b}$ then $\theta_{a}^{b}\left(\left\{x_{i}\right\}_{i \leqq b}\right)=\left\{x_{i}\right\}_{l \leqq a}$. For notational convenience, if $b$ is a limit ordinal let $M_{b-1}$ denote the space $L_{b}\left(M_{b}^{\prime}\right)$ and let $\mathbf{P}_{b-1}$ denote the function $f \circ \mathbf{P}_{b}$ where $f$ projects $L_{b}\left(M_{b}^{\prime}\right) \times[0,1]$ onto $L_{b}\left(M_{b}^{\prime}\right) \times\{0\}$.

Let $M=\lim \left\{M_{a}, \theta\right\}_{a<\alpha}$. If for each $a, I_{a}=M_{a-1} \times\{0\}$ then $M$ and the collection $\left\{I_{a}\right\}_{a<\alpha}$ satisfy the hypothesis of Theorem 1 because $M_{a}$ is irreducible from the point $B_{a}$ to each point of $I_{a}$. Thus $M$ is indecomposable. If $P \in M$ let $P_{\gamma}$ denote $\mathbf{P}_{\gamma}(P)$. Let $L$ denote the projection $L_{\alpha}$ as defined above.

Suppose $x$ is a point of $M$ and $w_{x}$ is the set to which $P$ belongs if and only if there exists a $\beta<\alpha$ so that if $\beta<\gamma<\alpha$ then $\pi_{a}\left(P_{\gamma}\right)=\pi_{a}\left(x_{\gamma}\right)$ for all $a$ so that $\beta<a \leqq \gamma$. Equivalently: $w_{x}$ is the point set to which $P$ belongs if and only if there exists a $\beta<\alpha$ so that $\pi_{a}(L(P))=\pi_{a}(L(x))$ for all $a>\beta$. The set $w_{x}$ will be shown to be the composant of $M$ containing $x$.

Suppose $P \in w_{x}$. Then there exists a $\beta<\alpha$ so that $\pi_{a}(L(P))=$ $\pi_{a}(L(x))$ for all $a>\beta$. Then $\left\{y \mid y \in M\right.$ and $\left(y_{\gamma}\right)_{a}=\left(x_{\gamma}\right)_{a}$ for all $a$ such that $\beta<a \leqq \gamma\}$ is a proper subcontinuum of $M$ containing $x$ and $P$. The following lemma implies that $w_{x}$ is a composant.

LEMMA A. If I is a proper subcontinuum of $M$ containing the point $x$ then there exists a $\beta<\alpha$ so that if $\beta<\gamma<\alpha$ then $\pi_{a}\left(\mathbf{P}_{\gamma}(I)\right)=\pi_{a}\left(x_{\gamma}\right)$ for all $a$ so that $\beta<a \leqq \gamma ;\left(\right.$ or, there exists $a \beta<\alpha$ so that $\pi_{a}(L(I))=$ $\pi_{a}(L(x))$ for all $a$ so that $\beta<a<\alpha$.)

Proof. Suppose that $I$ is a subcontinuum of $M$ containing the point $x$. Then there exists an element $\beta<\alpha$ so that $\mathbf{P}_{\beta}(I) \neq M_{\beta}$. Suppose that 
the lemma is false. Then there exists a first element $a_{1}>\beta$ so that $\pi_{a_{1}}(L(I))$ is non-trivial. Likewise there is a first element $a_{2}$ after $a_{1}$ and a first element $a_{3}$ after $a_{2}$ so that $\pi_{a_{2}}(L(I))$ and $\pi_{a_{3}}(L(I))$ are non-trivial, $\beta<a_{1}<a_{2}<a_{3}$.

Let $\gamma>a_{3}$. Suppose $0 \in \pi_{a_{i}}\left(\mathbf{P}_{\gamma}(I)\right)$ for some $i=1,2,3$. Then there is a number $t$ distinct from 0 in $\pi_{a_{i}}\left(\mathbf{P}_{\gamma}(I)\right)$. But $\mathbf{P}_{\gamma}(I)$ intersects $M_{a_{i}-1} \times\{0\}$ and $M_{a_{i}}-\left(M_{a_{i}-1} \times\{0\}\right)$, so $\boldsymbol{M}_{a_{i}-1} \times\{0\} \subset \mathbf{P}_{a_{i}}(I)$. Thus $M_{\beta} \subset$ $\mathbf{P}_{\beta}(I)$ which is a contradiction.

Suppose $1 \in \pi_{a_{2}}\left(\mathbf{P}_{\gamma}(I)\right)$. Then there is a number $t<1$ in $\pi_{a_{2}}\left(\mathbf{P}_{\gamma}(I)\right)$. But there is a number $r \geqq t$ so that $\left\{A_{a_{2}-1}\right\} \times[r, 1] \subseteq \mathbf{P}_{a_{2}}(I)$, this follows from the construction of $M_{a_{2}}$. Then $0 \in \pi_{a_{1}}\left(\mathbf{P}_{a_{2}}(I)\right)$ since $A_{a_{2}-1}=\{0\}_{i<a_{2}}$ and this is a contradiction. So $1 \notin \pi_{a_{2}}\left(\mathbf{P}_{\gamma}(I)\right)$. Similarly $1 \notin \pi_{a_{3}}\left(\mathbf{P}_{\gamma}(I)\right)$.

Suppose $0<t_{1}<t_{2}<1$ and $\left[t_{1}, t_{2}\right] \subset \pi_{a_{3}}\left(\mathbf{P}_{\gamma}(I)\right)$. But $\mathbf{P}_{a_{3}}(I)$ does not intersect any of the sets $\left\{\left\{A_{a_{3}-1}\right\} \times[1 /(2 i+2), 1 /(2 i+1)]\right\}_{i=0}^{\infty}$ or any of the sets $\left\{\left\{B_{a_{3}-1}\right\} \times[1 /(2 i+1), 1 / 2 i]\right\}_{i=1}^{\infty}$, or else either 0 or 1 would belong to $\pi_{a_{2}}\left(\mathbf{P}_{a_{2}}(I)\right)$. Then $\mathbf{P}_{a_{3}}(I)$ must be a subset of $M_{a_{3}} \times\{1 / k\}$ for some integer $k>1$. But $\pi_{a}\left(\mathbf{P}_{a_{3}}(I)\right)=\pi_{a}\left(\mathbf{P}_{\gamma}(I)\right)$ for $a \leqq a_{3}$ so $\pi_{a_{3}}\left(\mathbf{P}_{\gamma}(I)\right)=1 / k$ which is a contradiction. So the lemma must be true.

LEMma B. Suppose $q$ is a cardinal number and $\alpha$ is the first ordinal number so that $q=|\alpha|$. Then there exists a collection $G$ of functions from $\alpha$ into the set $\{0,1\}$ of cardinality $2^{q}$ so that if $f$ and $g$ belong to $G$ then the set $\{x \mid x \in \alpha$ and $f(x) \neq g(x)\}$ is cofinal in $\alpha$.

Proof. Let $T$ be a bijection from $\alpha \times \alpha$ onto $\alpha$. If $a \in \alpha$ then the set $T(\{a\} \times \alpha)$ is cofinal in $\alpha$. Suppose that $S$ is a subset of $\alpha$, let $f_{s}$ be the function from $\alpha$ into $\{0,1\}$ so that $f_{S}(t)=1$ if and only if $t \in T(S \times \alpha)$. Let $G=\left\{f_{s} \mid S\right.$ is a subset of $\left.\alpha\right\}$. Suppose $S_{1}$ and $S_{2}$ are two distinct subsets of $\alpha$ and $a$ is an element of $S_{1}$ not in $S_{2}$. Then $f_{S_{1}}(T(\{a\} \times \alpha))=1$ and $f_{S_{2}}(T(\{a\} \times \alpha))=0$ so $\left\{x \mid x \in \alpha\right.$ and $\left.f_{S_{1}}(x) \neq f_{S_{2}}(x)\right\}$ contains the set $T(\{a\} \times \alpha)$ which is cofinal in $\alpha$. Thus $|G|=2^{a}$ and the lemma is proven.

The continuum $M$ was constructed so that every function from $\alpha$ into the set $\{0,1\}$ belongs to $L(M)$. If $q$ is a cardinal number and $\alpha$ is the first ordinal number so that $q=2^{|\alpha|}$ then, by Lemma $\mathrm{B}$, the number of composants of $M$ is at least $2^{|\alpha|}$. If $c$ denotes the cardinality of $[0,1]$ then $M$ has cardinality at most $c^{|\alpha|}$. But $2^{|\alpha|}=c^{|\alpha|}$, so $M$ has $2^{|\alpha|}$ composants.

Notation: If $\lambda$ is a limit ordinal let $M_{\lambda}$ denote the indecomposable continuum obtained by the construction of Theorem 2 with $\lambda=\alpha$.

COROllary 2.1. If $X$ is a continuum then $X$ is homeomorphic to a retract of an indecomposable continuum with an arbitrarily large number of composants. 
Proof. It follows from the construction in [3] that $X$ is homeomorphic to a retract of an irreducible continuum $Y$. Then if $Y$ is irreducible from the point $A$ to the point $B$ merely replace $M_{0}$ by $Y$ and $\{0\}$ and $\{1\}$ by $A$ and $B$ respectively in the above construction.

COROLlaRy 2.2. There exists a non-metric continuum each proper subcontinuum of which is metric.

Proof. Consider $M_{\omega_{1}}$, where $\omega_{1}$ is the first uncountable ordinal. By Lemma $\mathrm{A}$, if $I$ is a proper subcontinuum of $M$ there is a point $x \in M$ and an element $\beta<\omega_{1}$ so that $\pi_{a}(L(I))=\pi_{a}(x)$ for all $a$ so that $\beta<a<$ $\omega_{1}$. Thus $L(I)$ is embedded in $\prod_{a \leqq \beta}[0,1]_{a} \times\left(\left\{\pi_{a}(L(x))\right\}_{a<\beta}\right)$. So $I$ is homeomorphic to a subset of the cartesian product of countably many intervals and hence is metric. For each $a<\omega_{1}$ let $x_{a}$ be the point of $\Pi_{l<\omega}[0,1]_{l}$ which is 1 at the ath coordinate and is 0 elsewhere. Then the set $\left\{x \mid x=x_{a}, a<\alpha\right\}$ is an uncountable set of points in $L(M)$ which contains none of its limit points. Thus $L(M)$ is not metric.

Observation 1. If $X$ is a non-metric continuum and every proper subcontinuum of $X$ is metric then $X$ is indecomposable.

Observation 2. The continuum $M_{\omega_{1}}$ has $2^{\boldsymbol{N}_{1}}$ composants, and $c \leqq$ $2^{\boldsymbol{N}_{1}} \leqq 2^{c}$. Thus the continuum could have $c$ or $2^{c}$ composants depending on which axioms of set theory are assumed. It is also possible that neither equality holds.

COROLlaRy 2.3. There exists a continuum M every proper subcontinuum of which is less numerous than $M$.

Proof. Let $\alpha$ be the first ordinal number so that $2^{c}<2^{|\alpha|}$, where $c$ is the cardinality of the interval $[0,1]$. Then if $\beta<\alpha, 2^{|\beta|}<2^{|\alpha|}$. Consider the continuum $M_{\alpha}$ constructed above. $M_{\alpha}$ contains at least $2^{|\alpha|}$ points. By Lemma A, if $I$ is a proper subcontinuum of $M$ there exists a point $x \in M$ and an element $\beta<\alpha$ so that $\pi_{a}(L(I))=\pi_{a}(x)$ for all $a$ so that $\beta<a<\alpha$. Thus $L(I)$ is embedded in $\Pi_{a \leqq \beta}[0,1]_{a} \times\left(\left\{\pi_{a}(L(x))\right\}_{\beta<\alpha}\right)$. So $I$ has at most $c^{|\beta|}$ points and $c^{|\beta|} \leqq 2^{c}<2^{|\alpha|}$. Again observe that any continuum having this property must be indecomposable.

THEOREM 3. Suppose $q$ is a cardinal number, $\alpha$ is the first ordinal number so that $|\alpha|=q$, and $C$ is a composant of $M_{\alpha}$. If $H \subset C$ and $|H|<\alpha$ then $\bar{H} \subset C$.

Proof. Suppose $H \subset w_{x}$. It follows from the definition of $w_{x}$ that there exists a $\beta<\alpha$ so that if $P \in H$ then $\pi_{a}(L(P))=\pi_{a}(L(x))$ for all $a$ 
so that $\beta<a<\alpha$. Suppose $Q \in M-w_{x}$. Then there exists a $\delta>\beta$ so that $\pi_{\delta}(L(Q)) \neq \pi_{\beta}(L(x))$. Let $S_{\delta}$ be a region in $[0,1]_{\delta}$ containing $\pi_{\delta}(L(\theta))$ and not $\pi_{\delta}(L(x))$. Then $R=\left\{Z \mid \pi_{\delta}(Z) \in S\right\}$ is an open set in $L(M)$ containing $L(Q)$ but no point of $L(H)$. So $Q \notin \bar{H}$. So $\bar{H} \subset w_{x}$.

Definition. The subset $H$ of the Hausdorff space $X$ is said to be conditionally compact if and only if it is true that every infinite subset of $H$ has a limit point in $H$.

COROLlARY 3.1. There exists a conditionally compact indecomposable connected Hausdorff space with only one composant.

Proof. By Theorem 3 any composant of $M_{\omega_{1}}$ is such a space.

\section{REFERENCES}

1. D. P. Bellamy, Mappings of indecomposable continua, Proc. Amer. Math. Soc., 30 (1971), 179-180.

2. S. Eilenberg and N. Steenrod, Foundations of Algebraic Topology, Princeton University Press, Princeton, New Jersey, 1952.

3. G. R. Gordh, Jr., Every continuum is a retract of some irreducible indecomposable continuum, Colloquia Mathematica Societaties Janos Bolyai, 8 (1972), 347-340.

4. R. L. Moore, Foundations of point set theory, Amer. Math. Soc. Colloq. Pub. XIII, Revised edition, Providence R. I., 1962.

Received June 23, 1975 and in revised form October 31, 1975.

AUBURn UnIVERSITY 



\section{PACIFIC JOURNAL OF MATHEMATICS}

\section{EDITORS}

RICHARD ARENS (Managing Editor)

University of California

Los Angeles, California 90024

\section{J. Dugundu}

Department of Mathematics University of Southern California Los Angeles, California 90007

D. Gilbarg and J. Milgram Stanford University Stanford, California 94305

\section{ASSOCIATE EDITORS}
E. F. BECKENBACH
B. H. NeumanN
F. WOLF
K. YoSHIDA

\section{SUPPORTING INSTITUTIONS}

UNIVERSITY OF BRITISH COLUMBIA CALIFORNIA INSTITUTE OF TECHNOLOGY

UNIVERSITY OF CALIFORNIA

MONTANA STATE UNIVERSITY

UNIVERSITY OF NEVADA

NEW MEXICO STATE UNIVERSITY

OREGON STATE UNIVERSITY

UNIVERSITY OF OREGON

OSAKA UNIVERSITY

\author{
UNIVERSITY OF SOUTHERN CALIFORNIA \\ STANFORD UNIVERSITY \\ UNIVERSITY OF HAWAII \\ UNIVERSITY OF TOKYO \\ UNIVERSITY OF UTAH \\ WASHINGTON STATE UNIVERSITY \\ UNIVERSITY OF WASHINGTON \\ AMERICAN MATHEMATICAL SOCIETY
}

The Supporting Institutions listed above contribute to the cost of publication of this Journal, but they are not owners or publishers and have no responsibility for its contents or policies.

Mathematical papers intended for publication in the Pacific Journal of Mathematics should be in typed form or offset-reproduced (not dittoed), double spaced with large margins. Underline Greek letters in red, German in green, and script in blue. The first paragraph or two must be capable of being used separately as a synopsis of the entire paper. Items of the bibliography should not be cited there unless absolutely necessary, in which case they must be identified by author and Journal, rather than by item number. Manuscripts, in duplicate, may be sent to any one of the four editors. Please classify according to the scheme of Math. Reviews, Index to Vol. 39. All other communications should be addressed to the managing editor, or Elaine Barth, University of California, Los Angeles, California, 90024.

100 reprints are provided free for each article, only if page charges have been substantially paid. Additional copies may be obtained at cost in multiples of 50 .

The Pacific Journal of Mathematics is issued monthly as of January 1966. Regular subscription rate: $\$ 72.00$ a year (6 Vols., 12 issues). Special rate: $\$ 36.00$ a year to individual members of supporting institutions.

Subscriptions, orders for back numbers, and changes of address should be sent to Pacific Journal of Mathematics, 103 Highland Boulevard, Berkeley, California, 94708.

PUBLISHED BY PACIFIC JOURNAL OF MATHEMATICS, A NON-PROFIT CORPORATION

Printed at Jerusalem Academic Press, POB 2390, Jerusalem, Israel.

$$
\begin{gathered}
\text { Copyright } 1976 \text { Pacific Journal of Mathematics } \\
\text { All Rights Reserved }
\end{gathered}
$$




\section{Pacific Journal of Mathematics}

Vol. 62, No. 2

February, 1976

Allan Russell Adler and Catarina Isabel Kiefe, Pseudofinite fields, procyclic

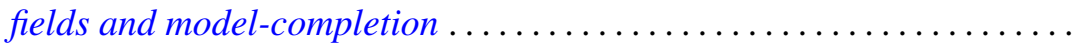

Christopher Allday, The stratification of compact connected Lie group

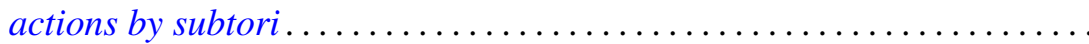

Martin Bartelt, Commutants of multipliers and translation operators .......

Herbert Stanley Bear, Jr., Ordered Gleason parts ..................

James Robert Boone, On irreducible spaces. II .....................

James Robert Boone, On the cardinality relationships between discrete

collections and open covers ............................

L. S. Dube, On finite Hankel transformation of generalized functions .......

Michael Freedman, Uniqueness theorems for taut submanifolds . . . . . . . . .

Shmuel Friedland and Raphael Loewy, Subspaces of symmetric matrices

containing matrices with a multiple first eigenvalue .............

Theodore William Gamelin, Uniform algebras spanned by Hartogs

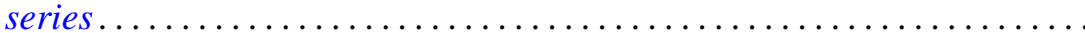

James Guyker, On partial isometries with no isometric part ............

Shigeru Hasegawa and Ryōtarō Satō, A general ratio ergodic theorem for

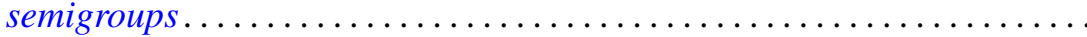

Nigel Kalton and G. V. Wood, Homomorphisms of group algebras with norm less than $\sqrt{2}$.

Thomas Laffey, On the structure of algebraic algebras...

Will Y. K. Lee, On a correctness class of the Bessel type differential operator $S_{\mu}$

Robert D. Little, Complex vector fields and divisible Chern classes ....

Kenneth Louden, Maximal quotient rings of ring extensions . .

Dieter Lutz, Scalar spectral operators, ordered $l^{\rho}$-direct sums, and the

counterexample of Kakutani-McCarthy . .

Ralph Tyrrell Rockafellar and Roger Jean-Baptiste Robert Wets, Stochastic

convex programming: singular multipliers and extended duality

singular multipliers and duality.

Edward Barry Saff and Richard Steven Varga, Geometric overconvergence of rational functions in unbounded domains ..........

Joel Linn Schiff, Isomorphisms between harmonic and P-harmonic Hardy

spaces on Riemann surfaces.

Virinda Mohan Sehgal and S. P. Singh, On a fixed point theorem of

Krasnoselskii for locally convex spaces.

Lewis Shilane, Filtered spaces admitting spectral sequence operations

Michel Smith, Generating large indecomposable continua . 